\title{
Design and Development of Efficient Cost Saving Algorithms for Guiding Customer Purchasing Patterns in Modern Consumerism Scenario Using Fuzzy Logic System
}

\author{
Sonia Maria D’Souza*, Dr. K Satyanarayan Reddy \\ Research Centre, Department of CSE, Cambridge Institute of Technology (affiliated to VTU, Belagavi), \\ Bangalore, Karnataka, India \\ Email: \{soniapradeepkumar, satyanarayan.reddy\}@ gmail.com
}

\begin{abstract}
Article Info
Volume 8 Issue 1

Page Number : 4 147-154

Publication Issue :

January-February-2021
\end{abstract}

\section{Article History}

Accepted : 01 Feb 2021

Published : 09 Feb 2021

\section{ABSTRACT}

This research deals with the development of a new architecture. The system deals with self-scanning and self-checkout of the consumer products. Here we have made an effort to take the system is supported by an app. The database is maintained in the local server and both mobile and the local host are connected to the same network. As a result, the user can use all the features of this system and send and receive the data which is required by the user. This framework takes care of taxations such as GST. The concept involves designing and implementation of a cost saving algorithms by using fuzzy logic system by using python programming for guiding customer purchasing patterns, retrieval and faster method of accessibility with respect to different patterns.

Keywords : Neurons, Fuzzy Logic System, QR Code, Bar Code, Servers, Database Systems, Images.

\section{INTRODUCTION}

Most of the Indian metropolitan cities have IT industries and millions of people have settled down from different places due to availability of various job opportunities, family, education and for many other reasons where ever they stay shopping has become routine. An app that helps these people to easy shopping is the e-Commerce app or the websites [1][2]. But these have few limitations as it takes more time for delivery and sometimes out of stock, No assurance of the product and many more. And when people go to shopping malls they enjoy shopping but get frustrated in the billing counter due to long queues. This work suggests "Queue-less Shopping Cart with Self-Checkout" (Qless) System. This research deals with the development of a new architecture. The system deals with self-scanning and self-checkout. The user needs to go through series of steps to use the major features of the system as authentication i.e., the username as unique name and a password [3][4]. Before the user undergoes the process of authentication he/she can register to the system and then login. This system is a very handy app based system guiding people about the routes to the mall and due consideration on ensured budget. Here all the data is stored in the cloud server and based on the input given by the user the data is fetched from it and displayed to the user by using efficient algorithm using fuzzy system[13]. The database is maintained in the local server and both mobile and the local host are connected to the same 
network. As a result, the user can use all the features of this system and send and receive the data which is required by the user. Since keep up the nearby server the portable that the client utilizes must be conveyed at first and afterward the client can use whatever number as could reasonably be expected.

\section{LITERATURE REVIEW}

An ANN is based on a collection of connected units or nodes called artificial neurons, which loosely model the neurons in a biological brain. Each connection, like the synapses in a biological brain, can transmit a signal to other neurons. An artificial neuron that receives a signal then processes it and can signal neurons connected to it. The "signal" at a connection is a real number, and the output of each neuron is computed by some non-linear function of the sum of its inputs. The connections are called edges. Neurons and edges typically have a weight that adjusts as learning proceeds. The weight increases or decreases the strength of the signal at a connection. Neurons may have a threshold such that a signal is sent only if the aggregate signal crosses that threshold. Typically, neurons are aggregated into layers. Different layers may perform different transformations on their inputs. Signals travel from the first layer (the input layer), to the last layer (the output layer), possibly after traversing the layers multiple times. [9][10][12]. During the learning procedure frame mind collection required. Ideal models of administered learning incorporate blunder adjustment learning, fortification enlightens and stochastic enlightens. A significant mistake assembly, for example the derogate of omission betwixt ideal and sort out constituent. Bespeak is to poll a lot loads which limit overestimate [5][6].

Interpret in particular technique, which is rudimentary to many intellect ideal models, Least Mean Square (LMS)) assembly. Solo learning uses farthest instructor be contingent on just quarter data. Moreover, implied patterned-relationship, makes data showed to the framework and distinguishes their 1 properties [7][8]. Ideal models of solo learning are Hebbian enlighten. Hebbian enlightens mainly focuses on network dynamics and neuron model. Let Mcoll be the number of collided slots in a frame after a round. The estimated number of the tags $(n)$ is then calculated by,

$$
=2.3922 * l l
$$

The underlying conjecture of this study is that neuro fuzzy systems should be constructed on a basis of simple processing units-fuzzy (logic) neurons whose transparency and learning abilities are accentuated to the highest possible extent. This would assure us that the resulting constructs will directly benefit from these features that will manifest in the overall network. The notion of logic (two-valued logic) has been broadly exploited as the sound basis for Boolean networks. On the other hand, the concept of fuzzy logic and logic processing seems to be far less exploited in this setting.

The primordial objectives of this study are fourfold [14].

- The revisit and systematize the array of the existing fuzzy logic neurons (fuzzy neurons, for short) with respect to their functionality, underlying logic, interpretation aspects and learning abilities. These are well documented in the existing literature but still require some systematization and their "readability."

- To develop architectures of fuzzy neural networks based on different fuzzy neurons. As the systems of such character are inherently heterogeneous, their functionality could be quite diversified and a suitable arrangement of the neurons in successive layers could result in a surprisingly rich collection of logic expressions and nonlinear characteristics of the neural mappings.

- To discuss various schemes of the development of the net- works with a special emphasis put toward the structural aspects of learning and its realization in terms of genetic optimization. 
- To discuss interpretability of fuzzy networks and introduce means of their effective readability through pertinent pruning mechanisms.

\section{BASIC TYPES OF LOGIC NEURONS}

The underlying taxonomy involves aggregative neurons and very much ties up to their logic functionality [15]. Their names reflect the underlying processing realized by the neurons. The aggregative neurons concentrate on the logic type of aggregation of the inputs (truth values).

\section{A. Aggregative Neurons}

Formally, these neurons realize a logic mapping from $[0,1]^{\mathrm{n}}$ to $[0,1]$. Two main classes of the processing units exist in this category [16]

OR neuron realizes an and logic aggregation of inputs $\left[\mathrm{x}_{1}, \mathrm{x}_{2}, \ldots . . \mathrm{x}_{\mathrm{n}}\right]$ with the corresponding connections (weights) $\mathrm{W}=\left[\mathbf{w}_{1}, \mathbf{w}_{2}, \ldots . . \mathbf{w}_{\mathbf{n}}\right]$ and then summarizes the partial results in an or-wise manner (hence, the name of the neuron). The concise notation underlines this flow of computing, OR $(\mathbf{x} ; \mathbf{w})$ while the realization of the logic operations gives rise to the ex- pression (referred to as an $\mathrm{s}-\mathrm{t}$ combination).

$$
\mathrm{Y}=\mathrm{S}_{\mathrm{i}=1}^{\mathrm{n}}\left(\mathrm{x}_{\mathrm{i}} \mathrm{tw} \mathrm{w}_{\mathrm{i}}\right) .
$$

The two essential operators used in the composition are the $\mathrm{t}$ - and $\mathrm{s}$-norms. Let us recall that by $\mathrm{t}$-norms we mean an and type of logic connective used to aggregate two fuzzy sets. The commonly used examples of t-norms include minimum, product, and Lukasiewicz and connective. The typical examples of s-norms (that are realizations of or logic connectives) involve maximum, probabilistic sum $(\mathrm{a}+\mathrm{b}-\mathrm{ab})$, and Lukasiewicz or connective. Bearing in mind the interpretation of the logic connectives $\left(t-\right.$ and $s^{-}$ norms), the OR neuron realizes the following logic expression being viewed as an underlying logic description of the processing of the input signals $\left(\mathrm{x}_{1}\right.$ and $\left.\mathrm{w}_{1}\right)$ OR $\left(\mathrm{x}_{2}\right.$ and $\left.\mathrm{w}_{2}\right) \ldots \ldots$. OR $\left(\mathrm{x}_{\mathrm{n}}\right.$ and $\left.\mathrm{w}_{\mathrm{n}}\right)(2)$

Apparently the inputs are logically "weighted" by the values of the connections before producing the final result in other words, we can treat " $y$ " as a truth value of the above statement where the truth values of the inputs are affected by the corresponding weights. Noticeably, lower values of discount the impact of the corresponding inputs; higher values (especially those being positioned close to 1) do not affect the original truth values of the inputs resulting in the logic formula. In limit, if all connections $\mathrm{w}_{\mathrm{i}}, \mathrm{i}=1$ to $\mathrm{n}$ then the neuron produces a plain or-combination of the in- puts, $\mathrm{y}=\mathrm{x} 1$ or $\mathrm{x} 2 \ldots . .$. or $\mathrm{xn}$. The values of the connections set to zero eliminate the corresponding inputs. Computationally

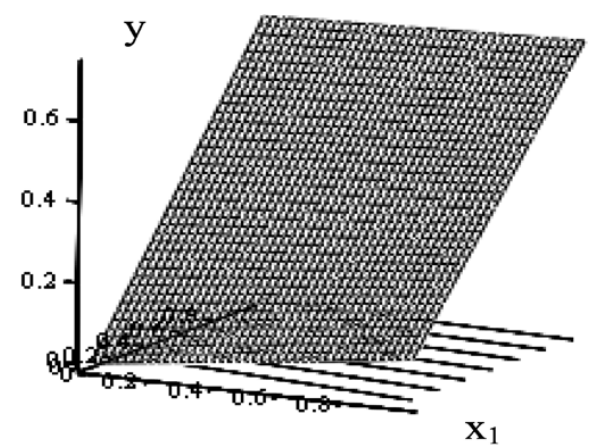

ORplot, ORplot

(a)

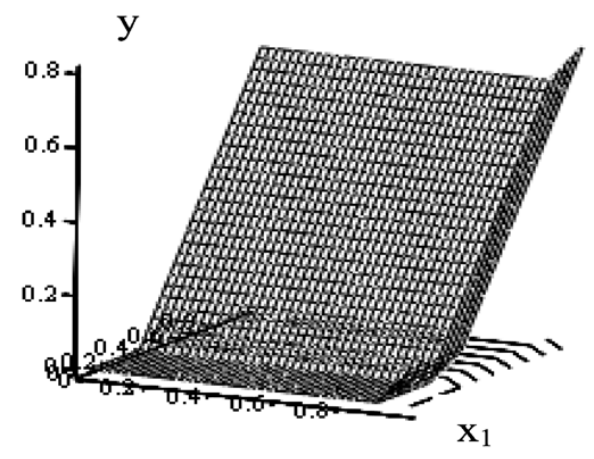

ORplot, ORplot

(b)

Figure 1 : Characteristics of the OR neuron for selected pairs of $\mathrm{t}$ - and $\mathrm{s}$-norms. In all cases, the corresponding connections are set to 0.1 and 0.7 with intent to visualize their effect on the inputoutput characteristics of the neuron. Product and 
probabilistic sum. (b) Lukasiewicz and and or connectives.

The OR neuron exhibits nonlinear characteristics (that is inherently implied by the use of the $\mathrm{t}$ - and $\mathrm{s}$ norms that are evidently nonlinear mappings). The plots of the characteristics of the OR neuron shown in Fig. 1 visualize this effect (note that the characteristics are affected by the use of some norms). The connections of the neuron contribute to its adaptive character; the changes in their values form the crux of the parametric learning.

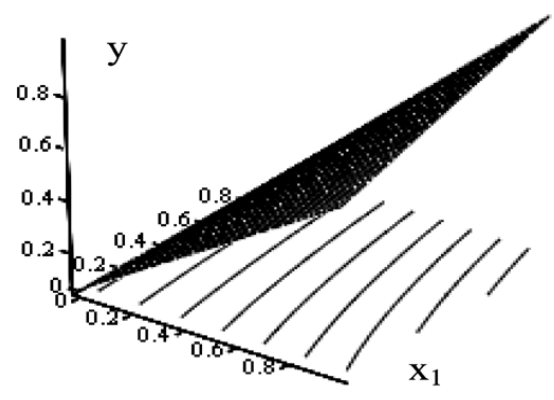
ANDplot, ANDplot

(a)

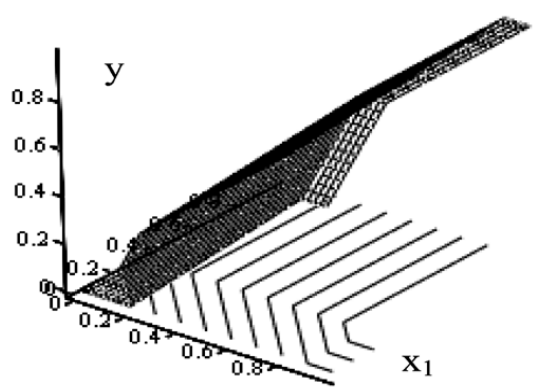

ANDplot, ANDplot

(b)

Figure 2 : Characteristics of AND neurons for selected pairs of $\mathrm{t}$ - and $\mathrm{s}$-norms. In all cases, the connections are set to 0.1 and 0.7 with intent to visualize their effect on the characteristics of the neuron. (a) Product and probabilistic sum. (b)Lukasiewicz logic connectives.

AND neuron The neurons in the category, denoted by $\mathrm{y}=\operatorname{AND}(\mathbf{x} ; \mathbf{w})$ with $\mathrm{x}$ and $\mathrm{y}$ being defined as in case of the OR neuron, are governed by the expression [15][16].

$$
\mathrm{Y}=\mathrm{T}_{\mathrm{i}=1^{\mathrm{n}}}(\mathrm{x} ; \text { swi })
$$

Here the or and and connectives are used in a reversed order. First, the inputs are combined with the use of the s-norm and the par- tial results are aggregated and-wise. Higher values of the connections reduce impact of the corresponding inputs. In limit $\mathrm{w}_{\mathrm{i}=1}$ eliminates the relevance of $\mathrm{xi}$. With all Wi set to 0 , the output of the AND neuron is just an and aggregation of the inputs.

\section{$\mathrm{Y}=\mathrm{x}_{1}$ and $\mathrm{x}_{2}$ and $\mathrm{x}_{\mathrm{n}}$}

Obviously this could be a matter of convenience and some tradition inherited from neural networks where higher value of the connections reflects its more evident impact. The characteristics of the AND neuron are shown in Fig. 2; note the influence of the connections and the specific realization of the triangular norms on the mapping completed by the neuron.

Let us conclude that the neurons are highly nonlinear processing units depending upon the specific realizations of the logic connectives. They also come with potential plasticity whose usage becomes critical when learning the networks involving these neurons.

\section{RELATIONSHIPS OF FUZZY NEURONS WITH FUZZY RELATIONAL EQUATIONS}

One can look at the fuzzy neurons discussed in the previous section from a slightly different perspective. The $\mathrm{s}-\mathrm{t}$ and $\mathrm{t}-\mathrm{s}$ calculations are in essence examples of so-called $s-t$ and $t$-s composition operators used in fuzzy sets. The inputs and connections can be treated as discrete n-dimensional fuzzy sets whose convolution is computed by means of the s- $t$ composition (OR neuron) and t-s composition (AND neuron). This simple observation links fuzzy neurons with the theory of fuzzy relational equations a wellestablished area of fundamental and applied pursuits in fuzzy sets. We can treat a fuzzy neuron 
as a realization of some fuzzy relational equation $\mathrm{OR}(\mathbf{x} ; \mathbf{w})$ or AND $(\mathbf{x} ; \mathbf{w})$.

Two fundamental problems are sought: (a) solving the equation with respect to for $\mathrm{x}$ and $\mathrm{y}$ given (usually referred in the theory of relational equations to as an estimation problem), and (b) solving the equation with respect to assuming that and $\mathrm{y}$ are provided (which refers to as an inverse problem).

In both cases, the theory provides us with interesting and general results. The first problem concerns the estimation of the connections of the neuron and is inherently tied to the learning of the network composed of logic neurons. Its generalized version involving solving a system of relational equations with a finite set of inputoutput pairs given is the standard version of the estimation problem. The inverse problem is focused on constructing inputs $(\mathbf{x})$ leading to the required output. The theory of fuzzy relational equations shows that in general there could be families (rather than unique solutions) to such equations, states how to effectively construct extreme (viz. maximal or minimal) solutions. These findings hold under a strong assumption that there is a nonempty family of solutions. Further- more the generality of the results is assured for some subset of $\mathrm{t}$ - and $\mathrm{s}$-norms used in the design of the composition operator; these solutions are obtained for max - $t$ composition and min -s convolution of fuzzy sets [16]. Interestingly, the generality of the solutions is not guaranteed for the general s- $t$ or t-s composition for any $\mathrm{t}-$ and s-norm. The relevance of the theoretical framework should be cast in a certain set- ting; in essence we can envision that the solutions can only be approximate and we can use them as a starting (initial) configuration of the connections the learning could start off. This is, in particular, quite relevant in case of gradient-based methods for which the choice of the starting point plays an essential role. The point worth mentioning here is that while relational equations form an important theoretical framework, they are not extremely well positioned to deal with problems of high dimensionality (in virtue of their logic framework they never intended to assume a leading role there). We relate to them in our discussion yet exploiting a different optimization environment of genetic algorithms, their role becomes more profound at the interpretation end along with some specialized systems obtained after pruning.

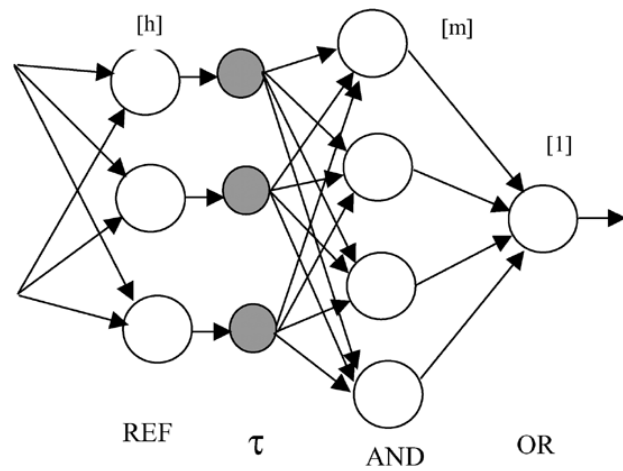

Figure 3 : General Architecture of the Network Constructed with Logic-based Neurons

\section{INTERFACES OF FUZZY NETWORKS}

In a nutshell, the fuzzy networks complete a logicbased processing of input signals and realizes a certain logic-driven map- ping between input and output spaces. As they interact with a physical world whose manifestation does not usually arise at the level of logic (multivalued) signals, it becomes apparent that there is a need for some interface of the model. Such interfaces are well known in fuzzy modeling [17 ]. They commonly arise under a name of fuzzifiers (granular encoders) and defuzzifiers (granular decoders). The role of the encoder is to convert a numeric input coming from the external environment into the internal format of membership grades of the fuzzy sets defined for each input variable. These results of a nonlinear normalization of the input (no matter what original ranges the input variables assume) and a linear increase of the dimensionality of the new logic space in 
comparison with the original one). The decoder takes the results of the logic processing and transforms them into some numeric values. The layered architecture of the fuzzy models with clearly distinguished interfaces and the logic-processing core is illustrated in Fig. 4. With the design of the interfaces, we exercise two general approaches.

1) Granulation of individual variables: This mechanism of granulation is quite common in the realm of fuzzy modeling. In essence, we define several fuzzy sets in the universe of discourse of the variable of interest so that any input is transformed via the membership functions defined there and the resulting membership grades are used in further computations by the model.

From the design standpoint, we choose a number of fuzzy sets, type of membership functions and a level of overlap between consecutive fuzzy sets. Some general tendencies along this line are thoroughly reported in the literature. By selecting the number of fuzzy sets (usually between 3 and 9), we position modeling activities at some required level of information granularity (a level of modeling details we are interested in). The type of membership functions helps model the semantics of the information granules. Among many possibilities, we commonly encounter tri- angular fuzzy sets and Gaussian membership functions. These two types come with an extensive list of arguments that help make a suitable selection with respect to the main objectives of the model (e.g those concerning a tradeoff between interpretation and accuracy of modeling). The overlap level is essential from different points of view, namely a) semantics of the linguistic terms b) nonlinear numeric characteristics of the fuzzy model, and c) completeness of the model.

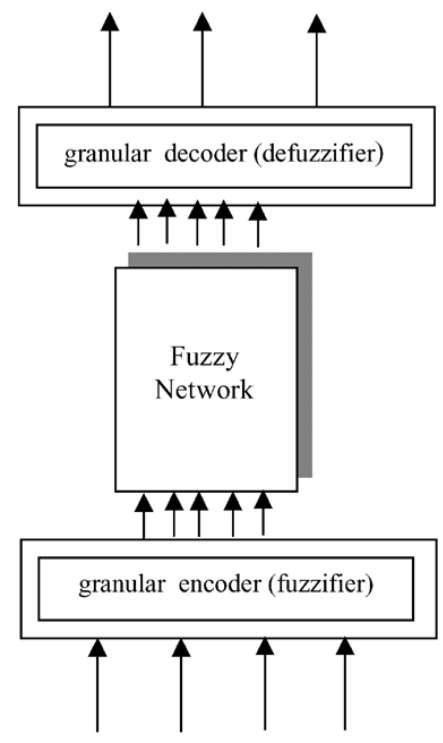

Figure 4 : General layered structure of fuzzy modeling. The use of granular encoders and decoders are essential in the development of communication

mechanisms with the modeling environment.

2) Nonlinear or linear normalization: Here we transform an original variable defined in some space, say $[a, b]$ (subset of $R$ is scaled to the unit interval. This could be done with the aid of some mapping $\phi$ $:[\mathrm{a}, \mathrm{b}]-[0,1]$. The mapping may be chosen to be linear (with some ramping effect) or nonlinear. In any case, we consider that $\$ \backslash$ phi $\$$ is monotonically increasing with the boundary conditions $\phi(\mathrm{a})=0[7]$.

\section{RESULTS AND DISCUSSIONS}

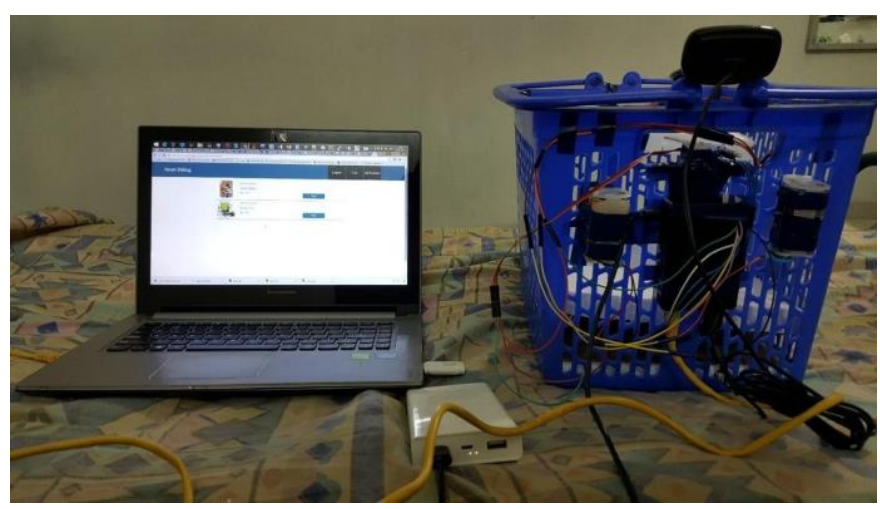

Figure 6. Prototype of shopping cart 


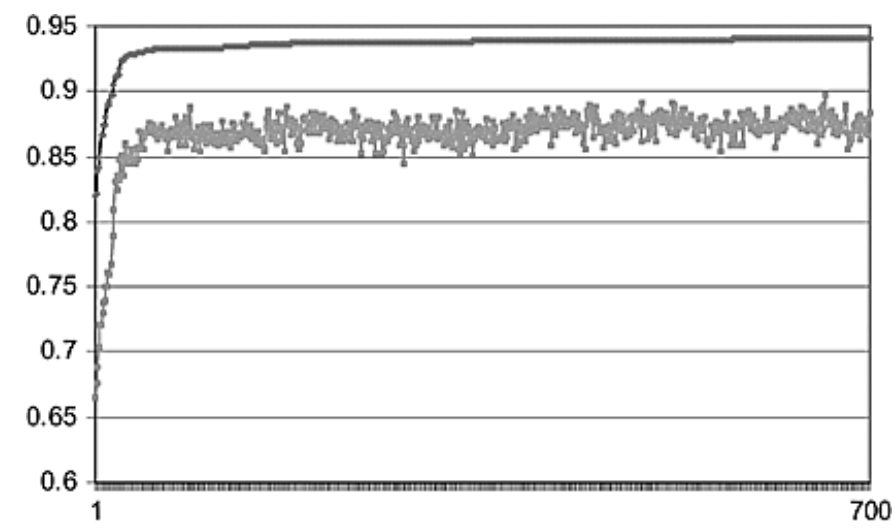

Figure 6. Fuzzy optimization of the network; fitness function (best and average) produced in successive generations of the data sets.

In the development of the network we use $60 \%$ of the dataset (training data) selected at random from the entire set with the rest used for testing purposes.

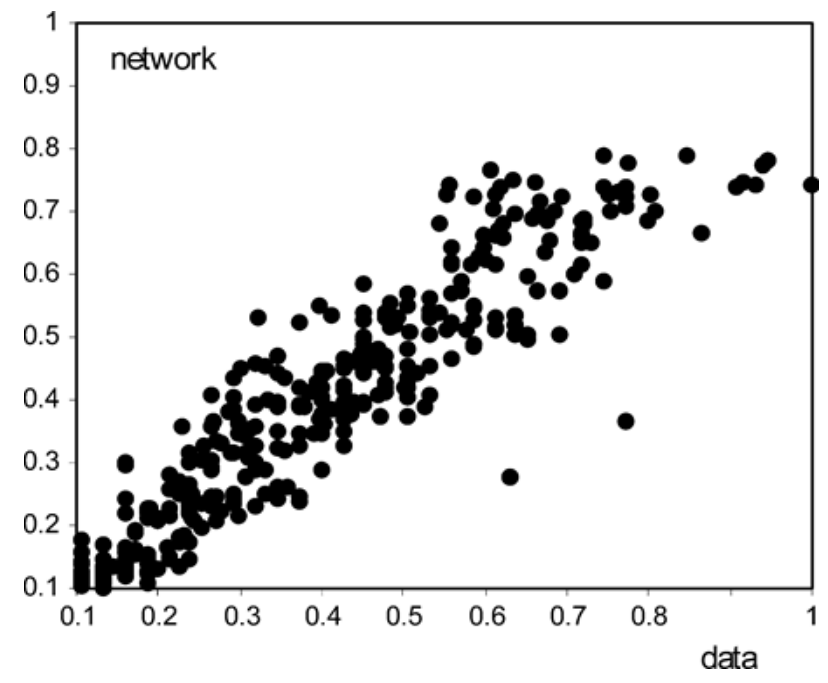

Figure 7. Network versus data. All data shown with a few discrepancies between the network and data

The overall performance of the network (shown for the entire dataset) is presented in Fig.7 with the experimental data visualized with respect to the corresponding outputs of the network and clarity of the image is dropped.

\section{CONCLUSION}

Interpretation mechanisms with an emphasis placed on the systematic pruning process that should be guided by some well-defined development criteria. The two potential candidates could involve a criterion of accuracy (which expresses how much the pruning affects the performance index) and a criterion of interpretability. This one could be more difficult to interpret and attach a tangible interpretation; as one among possible alternatives we can count the number of connections dropped and link it to the structural measure of complexity).The realization of the templates may trigger more research into hierarchical networks composed of a collection of specialized subnetworks. Time Complexity is more to process the data. Image collision is more.

\section{REFERENCES}

[1]. Zeeshan Ali, Reena Sonkusare, "RFID Based Smart Shopping and Billing", International Conference on Advances in Communication and Computing Technologies, IEEE Paper Publications, Vol 4,pp1-3,2014.

[2]. P.Chandrashekar,T.Sangeetha,"Smart Shopping trolley using RFID and ZigBee", International Conference on Information Communication and Embedded Systems, IEEE Paper Publications,Vol 4,PP 1-4,2014.

[3]. A.Asaduzaman, S.Mazumdar, S.Salinas,Md. F.Mridha, " A Security Aware Near Field Communication for Shopping Application", International Conference on Networking System And Security,IEEE Paper Publications, Vol 7, PP 33-38,2017.

[4]. Nethmi Deshani Hettiarachchi , Sobhani Umanga Pilapitiya, Nirmal Sankalpa Jayasinghe, Himash Deemantha, Sudheera Vitharana," I Shopping and Predicate Analysis System Using Data Mining", International Conference on Data Science and Data Intensive Systems, IEEE Paper Publications, Vol 5,PP 1-8,2015.

[5]. Zimu Zhou, Longfei Shangguan, Xiaolong Zheng, Lei Yang," Design and Implementaion of an RFID -Based Customer Shopping Behaviour Mining System", ACM transaction on 
Networking, IEEE Early Access Article,Vol 7,PP 1-14,2017.

[6]. Amine Karmouche, Yassine Salih-Alj, Jawad Abrache "Distributed Aisle-Level Scanning Approach for RFID Shopping Systems", International Conference on Logistics Operations and Management, IEEE Paper Publications, Vol 4, PP 1-5,2014.

[7]. Ruinian Li, Tianyi Song, Nicholas Capurso, Jiguo Yu, Jason Couture " IoT applications on Secure Smart Shopping System", IEEE Internet of Things Journal, Vol 7,pp 1-1,2017.

[8]. Xuan Liu, Haitao Zhang, Jingxian Fang, Guan Guan, Yundi Huang "Intelligent Shopping Cart with Quick Payment Based on Dynamic Target Tracking", International Conference on Cloud Computing and Intelligence Systems,IEEE Conference Publications, Vol 6, pp 88-93,2016.

[9]. Hsin-Han Chiang, Wan-Ting You, Shu-Hsuan Lin, Wei-Chih Shih, Yu-Te Liao, Jin-Shyan Lee, Yen-Lin Chen, " Development of Smart Shopping Carts with Customer-Oriented Service", International Conference on System Science and Engineering, IEEE Paper Publications, Vol 6, PP 1- 2,2016.

[10]. Xingguo Li, Tong Wang, Gaofeng Fan, Xun Wang, "Autonomic Customer Service System", International Conference on Data Science and Data Integrity, IEEE Paper Publications, Vol 6, PP 1-2,2016.

[11]. Dharm Singh Jat,Samiksha Shukla, Aynur Unal,Durgesh Kumar Mishra, "A Survey on Guiding Customer Purchasing Patterns in Modern Consumerism Scenario in India", appeared in Data Science and Security: Proceedings of IDSCS 2020, Published by Springer Lecture Notes in Network and Systems 132,https://doi.org/10.1007/978-981-15-53097_12(eBook).

[12]. Yu-Dong Zhang, Tomonoby Senjyu, Chakchai SO-IN and Amit Joshi, "Design and Development of Efficient Cost-Saving Algorithms for Guiding Customer Purchasing
Patterns in Modern Consumerism Scenario Using Feed Forward Back Propagation Neural Networks", appeared in Smart Trends in Computing and Communications: Proceedings of SmartCom 2020; Smart Innovation, Systems and Technologies, published by Springer Nature Singapore Pte Ltd.2021, Volume 182, pp.469-476. ISSN 2190-3026(electronic), ISBN 978-981-15-5224-3(eBook).

[13]. J. Casillas et al., Ed., Interpretability Issues in Fuzzy Modeling, Berlin, Germany: SpringerVerilog, 2003.

[14]. J. A. Dickerson and M. S. Lan, "Fuzzy rule extraction from numerical data for function proximation," IEEE Trans. Syst., Man, Cybern., vol. 26, pp. 119-129, Aug. 1995.

[15]. A. Di Nola, W. Pedrycz, and S. Sessa, "Fuzzy relational structures: The state of art," Fuzzy Sets Syst., vol. 75, pp. 241-262, 1995.

[16]. A. F. Gomez-Skarmeta, M. Delgado, and M. A. Vila, "About the use of fuzzy clustering techniques for fuzzy model identification," Fuzzy Sets Syst., vol. 106, pp. 179-188, 1999.

[17]. W. Pedrycz, "Approximate solutions of fuzzy relational equations, "Fuzzy Sets Syst., vol. 26, pp. 183-202, 1988.

\section{Cite this article as :}

Sonia Maria D'Souza, Dr. K Satyanarayan Reddy, "Design and Development of Efficient Cost Saving Algorithms for Guiding Customer Purchasing Patterns in Modern Consumerism Scenario Using Fuzzy Logic System", International Journal of Scientific Research in Science, Engineering and Technology (IJSRSET), Online ISSN : 2394-4099, Print ISSN : 2395-1990, Volume 8 Issue 1, pp. 147-154, January-February 2021. Available at doi : https://doi.org/10.32628/IJSRSET218127 Journal URL : http://ijsrset.com/IJSRSET218127 